\title{
Liquid Crystal Lens Array with Thermally Controllable Focal Length and Electrically Convertible Lens Type
}

\author{
Kyong Chan Heo, Jin Hyuk Kwon, and Jin Seog Gwag* \\ Department of Physics, Yeungnam University, 214-1 Dae-dong, Gyeongsan 712-749, Korea
}

(Received December 23, 2014 : revised February 2, 2015 : accepted February 4, 2015)

\begin{abstract}
This paper reports the fabrication of a lenticular liquid crystal (LC) lens array with thermally tunable focus and with the function of a convertible lens type, using the surface structure of a UV-curable polymer and a twisted-nematic (TN) LC cell. The TN LC cell makes the LC lenticular lens function as a converging or diverging lens by controlling electrically the polarization of input light. Therefore, the focal lengths for both the converging and diverging lenses, which can be switched from the TN cell, can be tuned by changing the effective refractive index of the LC by Joule heating of the transparent electrode. As a result, the focal length of the lens with the E7 LC was changed continuously from 8.7 to $31.2 \mathrm{~mm}$ for the converging lens type and from -9.8 to $-14.2 \mathrm{~mm}$ for the diverging lens when the temperature was increased from 25 to $56^{\circ} \mathrm{C}$.
\end{abstract}

Keywords : Lenticular lens array, Focal length tuning, Liquid crystal device, Dynamic lens

OCIS codes : (230.3720) Liquid-crystal devices; (120.2040) Displays; (130.0250) Optoelectronics

\section{INTRODUCTION}

Tunable-focus lens arrays have been reported by many researchers in various fields, such as optoelectronics, optical communications, high-density data storage, cellular-phone cameras, and integral-imaging information processing for various optical applications [1, 2], because controlling the focal length of a lens arrays plays an important role in its active optical performance. Several methods for controlling the radius of curvature of the lens have been proposed to obtain a tunable focusing lens, such as electrowetting, dielectrophoretic, and adaptive liquid microlenses based on isotropic media. On the other hand, it is difficult to tune precisely the curvature of the lenses. Therefore, the tunable-refractive-index method was suggested as an alternative. A range of techniques using the large anisotropy of LCs as another means to a tunable focusing lens have been investigated to fabricate tunable liquid crystal (LC) lens arrays including polymer structure and an LC modulator, such as surface-relief profile, zonepatterned structure, and polymer network LC microlens arrays[3-5]. LC lenses have merits, such as light weight, no mechanical moving parts, and low cost. To adjust the focal length, most LC lenses change the refractive index by controlling the electric field distribution in the cell [3-15].

On the other hand, a complicated special structure is required to realize a continuous-gradient refractive index distribution with an electric field distribution. Moreover, tuning continuously the focal points with an electric field in a curved patterned structure is difficult, owing to the partial distortion of the electric field in a curved structure, which can generate LC deformation. Therefore, it is important to find a more reliable method for a continuously tunable objective distance.

This paper reports the fabrication of an LC lenticular cell with thermally tunable focusing, using the curvature structure of a UV-curable polymer, combined with a TN (twisted nematic) LC cell, along with their optical characteristics. The controllable focal length of the proposed LC lens is based mainly on the difference in refractive index between the curved polymer structure and LC, owing to the different physical properties of the $\mathrm{LC}$ molecules according to temperature. Here, in the proposed lenticular LC lens, the temperature can be varied by Joule heating according to the voltage applied to the transparent electrode in the LC lens cell. The TN cell switches a converging to a diverging lens and vice versa.

\footnotetext{
*Corresponding author: sweat3000@ynu.ac.kr

Color versions of one or more of the figures in this paper are available online.
} 


\section{OPERATING PRINCIPLE AND EXPERIMENTAL PREPARATION}

Figure 1 presents a schematic diagram and the operation principles of the proposed lenticular LC lens array and TN cell. As shown in Fig. 1(a), the structure is composed of an indium tin oxide (ITO) electrode deposited on a lenticular polymer array lens,-; an LC layer,-; a polarizer; and a TN cell. Bare glass without an ITO-electrode is used as the top substrate. Figures 1(b) and 1(c) outline the operational principles showing how the proposed LC lens array functions as converging and diverging lenses, respectively, through the "off" and "on" voltage states of the TN cell attached to the polarizer changing the focal length according to the temperature. Based on geometric optics, the focal length $\mathrm{f}$ of a microlens can be determined as follows: [16, 17]

$$
\mathrm{f}=\mathrm{R} /\left(\mathrm{n}_{\mathrm{LC}}-\mathrm{n}_{\mathrm{p}}\right)
$$

where $\mathrm{R}$ is the radius of curvature of the lens surface. Supposing spherical curvature, the radius of curvature $\mathrm{R}$ of a lenticular LC lens can be approximated as

$$
\mathrm{R}=\left(\mathrm{h}^{2}+\mathrm{W}^{2}\right) / 2 \mathrm{~h}
$$

as shown in Fig. 1(a), where $\mathrm{h}$ and $\mathrm{W}$ indicate the height and half width of a lenticular lens. $n_{L C}$ and $n_{p}$ are the effective refractive indices of the $\mathrm{LC}$ layer and the polymer lens, respectively. As shown in Figs. 1(b) and $1(\mathrm{c})$, the rubbing directions of the bottom and top substrates of the TN cell are respectively parallel and perpendicular, to the transmission axis of the polarizer. For the "off" state of the TN cell, the vibration direction of the linearly polarized light passing through the polarizer is rotated $90^{\circ}$ by the $\mathrm{TN}$ cell and the linearly polarized light is parallel to the major axis of the LC directors (the $\mathrm{n}_{e}$ direction) in the lens array. The effective refractive index of $\mathrm{E} 7 \mathrm{LC} \mathrm{n}_{L C}=1.739$ is larger than $\mathrm{n}_{p}=1.625$. Therefore, the lenticular LC lens cell works as a converging lens and the beam of light converges to a point, as shown in Fig. 1(b). On the other hand, for the "on" state of the TN cell, the LCs align vertically and the linearly polarized light from the polarizer then propagates through the TN cell without any change in polarization, because the vibration direction of the light coincides with the ordinary axis $\left(\mathrm{n}_{\mathrm{o}}\right.$ direction) of the LCs in the TN cell. Therefore, the linearly polarized light is parallel to the minor axis of the LC directors (the $\mathrm{n}_{o}$ direction) in the lens array. The effective refractive index of the E7 $\mathrm{LCn}_{L C}=1.522$ is then smaller than $\mathrm{n}_{p}$. As a result, this lenticular LC lens operates as a diverging lenticular

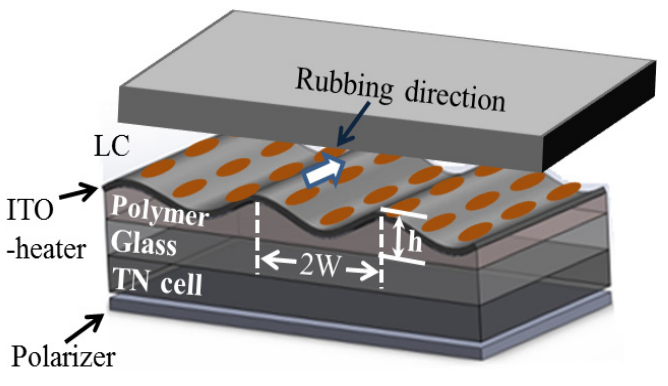

(a)

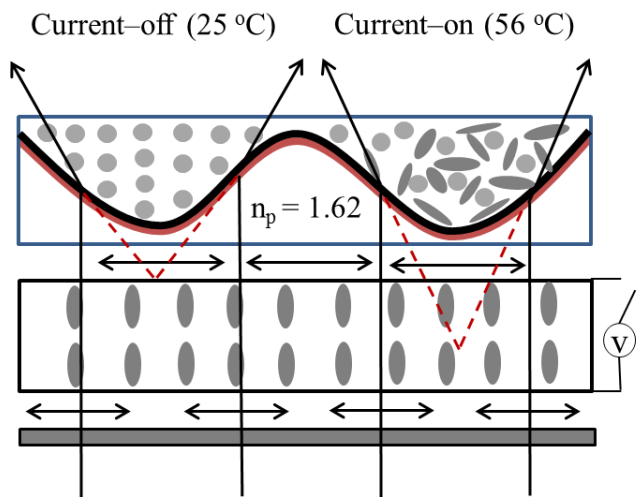

(c)

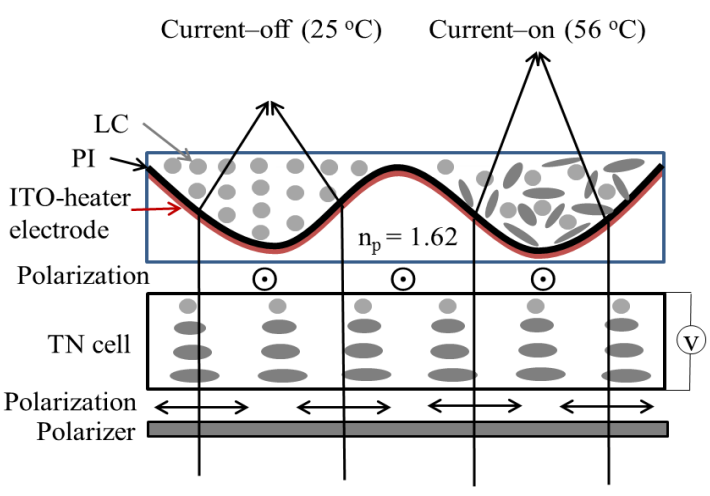

(b)

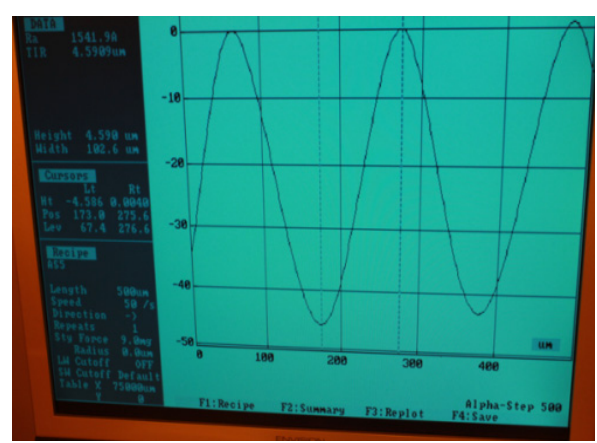

(d)

FIG. 1. (a) Schematic diagram of the proposed lenticular LC lens cell structure with a TN cell. Operating principle of the LC lens cell under (b) voltage "off"- state and (c) voltage "on"- state of the TN cell. (d) Image of the lenticular lens array measured by an Alpha step. The LC was aligned homogeneously on both surfaces. 
lens array, showing a negative focal length.

In addition, the refractive indices of the E7 LC were reported to depend on temperature. The temperature-dependent refractive indices of the $\mathrm{LC}$ can be expressed by Wu's model equation as follows [13]:

$$
\begin{aligned}
& \mathrm{n}_{\mathrm{e}} \approx \alpha^{-} \beta \mathrm{T}+(2 / 3) \Delta \mathrm{n}(0)\left(1-\mathrm{T} / \mathrm{T}_{\mathrm{c}}\right)^{\gamma} \\
& \mathrm{n}_{\mathrm{o}} \approx \alpha-\beta \mathrm{T}-(1 / 3) \Delta \mathrm{n}(0)\left(1-\mathrm{T} / \mathrm{T}_{\mathrm{c}}\right)^{\gamma}
\end{aligned}
$$

where $\gamma$ is a material parameter, $\Delta \mathrm{n}(0)=\mathrm{n}_{\mathrm{e}}(0)-\mathrm{n}_{\mathrm{o}}(0)$ is the birefringence of the $\mathrm{LC}$ at $\mathrm{T}=0 \mathrm{~K}$, and $\mathrm{T}_{\mathrm{c}}$ is the clearing temperature. Based on Wu's model of Eqs. (3) and (4), the characteristics of the temperature-dependent refractive indices of the E7 LC have been reported [12-15, $18,19]$. From the experimental result, the values of the constant terms for the temperature-dependent refractive indices are as follows: $\Delta \mathrm{n}(0)=0.3435, \gamma=0.1938, \alpha=$ 1.7432 , and $\beta=5 \times 10^{-4}\left(\mathrm{~K}^{-1}\right)$. The nematic-isotropic transition temperature of the $\mathrm{E} 7 \mathrm{LC}$ is $58^{\circ} \mathrm{C}$. In addition, Jun $\mathrm{Li}$ et al. examined the wavelength-dependent refractive indices of E7 LC [15]. Our experimental result was evaluated based on the characteristics of the temperature-dependent indices (at a wavelength of $560 \mathrm{~nm}$ ) of the E7 LC used in this lenticular lens, according to previous experiment result $[13,18]$.

To manufacture the lenticular lens structure, an ultraviolet (UV) curable-polymer (NOA 1625, Norland Products Inc.) was first spin-coated on a glass substrate at $4000 \mathrm{rpm}$ for $75 \mathrm{~s}$. The polymer layer was then exposed to UV light at $365 \mathrm{~nm}$ for $100 \mathrm{~s}$ under a lenticular-patterned photomask to fabricate a lenticular lens array. A second UV exposure was carried out for $30 \mathrm{~min}$ without a photomask for full curing of the surface lenticular polymer structure. The width $2 \mathrm{~W}$ and height $\mathrm{h}$ of the lenticular polymer lens were approximately 200 and 4.6, respectively. The characteristics of the lenticular lens array produced were observed using an Alpha step, as shown in Fig. 1(d), exhibiting a clear lens image. An ITO heater layer of approximately $70 \mathrm{~nm}$ was deposited on the surface of the lenticular lens array by RF magnetron sputtering. The electrical resistivity of the ITO layer deposited on the polymer lens array was approximately $3.5 \times 10^{-3} \Omega \cdot \mathrm{cm}$. Polyimide (PI), $80 \mathrm{~nm}$ in thickness, was spin-coated on the ITO as an LC alignment layer and baked for $5 \mathrm{~min}$ at $100^{\circ} \mathrm{C}$ to remove the solvent, followed by further baking at $200^{\circ} \mathrm{C}$ for $45 \mathrm{~min}$ to induce polymerization. Therefore, the bottom substrate was composed of a transparent ITO heater layer, a lenticular lens array on the glass substrate, and PI. The temperature of the lenticular LC lens cell can be tuned by proper control of the electrical current because the heating material is an ITO conductor with a proper resistance. A glass substrate coated with PI was used as the top substrate. The PI layers of the top and bottom substrates were rubbed parallel to each other to align the LCs.

The materials used were a commercial PI (AL-2001,
Nissan Chemical Co.) for the homogeneous alignment and a nematic LC (E7, Merck Chemical Co.). The ordinary and extraordinary refractive indices of the LC were $\mathrm{n}_{o}=$ 1.522 ande $=1.739(\Delta n=0.217)$ at room temperature, respectively. The cell thickness of the LC lens was maintained using 10-thick glass spacers and the $\mathrm{LC}$ was injected via capillary action.

In the case of the TN cell, the PI and LC employed were the same materials used for the LC lens array. The PI layers of the top and bottom substrates were rubbed perpendicular to each other to make a $90^{\circ}$-twisted LC structure. The thickness of the TN cell was maintained using 20 -thick glass spacers to satisfy Mauguin's condition (the limit of slow twist), $\varphi=<<\Gamma=2 \pi \Delta n d$. Under the conditions $\Delta n d$ $\approx 4.34$ for $\lambda=560 \mathrm{~nm}$ and twist angle $\varphi=\pi / 2\left(90^{\circ}\right), \Gamma$ was found to be 30 times higher than that of $\varphi$. Therefore, the Mauguin's condition is satisfied, and the linearly polarized light passing through the polarizer can be rotated precisely by $90^{\circ}$ at all visible wavelengths in the "off'-state. Furthermore, to block temperature effect propagating from the LC lens array, which may influence Mauguin's condition, we can put a thermally insulating material perhaps air between the TN cell and the LC lens array.

\section{RESULTS AND DISCUSSION}

Figure 2 shows the change in the focused-beam image from lenticular $\mathrm{LC}$ cells at room temperature $\left(25^{\circ} \mathrm{C}\right)$ with the "off" and "on" states of the TN cell inserted between the polarizer and LC lens cell. These reveal two methods to control the focusing characteristics of the proposed lenticular LC lens array. Figure 2(a) shows an optical microscopy image of a focused beam from the lens cell when the TN cell is in the "off" state. In this case, the polarization direction passing through the TN cell is parallel to the alignment direction of the lenticular LC lens cell. The effective refractive index $n_{L C}$ of the LC approximately becomes $n_{e}$ Therefore, the lenticular LC lens acts as a converging lens because $n_{L C}=\left(n_{e}=1.739\right)$ is larger than $n_{p}$ (1.62) (Fig. 2(a)). In addition, because the width $2 \mathrm{~W}$ and height $\mathrm{h}$ of the lenticular polymer lens were approximately 200 and 4.6, respectively, from Eq. (2), R was calculated to be approximately $1.10 \mathrm{~mm}$. Based on these results, the focal length was evaluated to be approximately $8.45 \mathrm{~mm}$ at room temperature $\left(25^{\circ} \mathrm{C}\right)$. Furthermore, the focal length measured at room temperature $\left(25^{\circ} \mathrm{C}\right.$ at $\left.0 \mathrm{~V}\right)$ was approximately matched at $8.7 \mathrm{~mm}$. In addition, when the TN cell is in the "on" state, the polarization after propagating through the $\mathrm{TN}$ cell is perpendicular to the rubbing direction of the $\mathrm{LC}$ lens cell and the effective index of LC becomes approximately $n_{o}=1.52$. Therefore, as $n_{o}$ $<n_{p}$, the lenticular LC lens acts as a diverging lenticular lens array, as shown in Fig. 2(b). Based on Eq. (1), the focal length was calculated to be approximately -10.29 $\mathrm{mm}$. This shows that the lens type can be converted by 
controlling the polarization direction of incident light using the TN cell.

To show an LC lens array acting as a dynamic converging lens, the focal images were observed at different temperatures when the TN cell was in the "off'-state. Figure 3 shows various optical microscope images of the beams focused

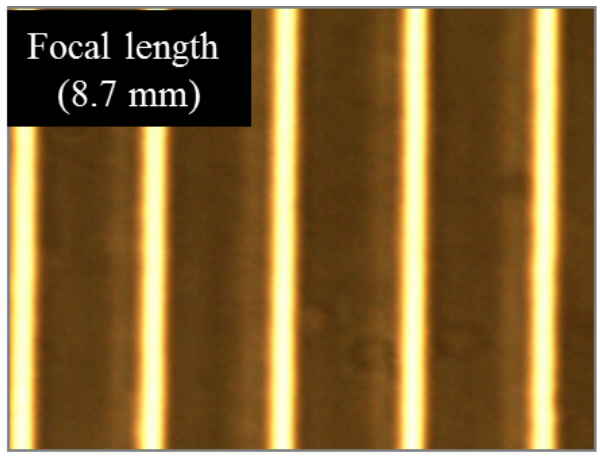

(a)

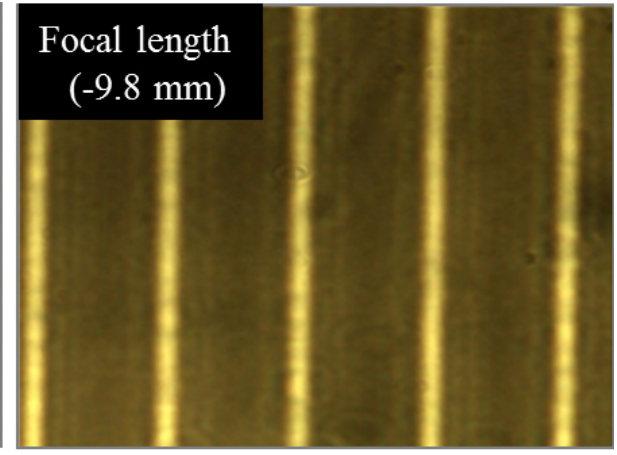

(b)

FIG. 2. Focusing images of the beam produced by the lenticular LC lens cell at room temperature with the "off" and "on" states of the TN cell inserted between a polarizer and LC lens cell. The vibration direction of the linearly polarized light is parallel to the major axis of the LCs in the LC lens array in (a), and is parallel to the minor axis of LCs in the LC lens array in (b).

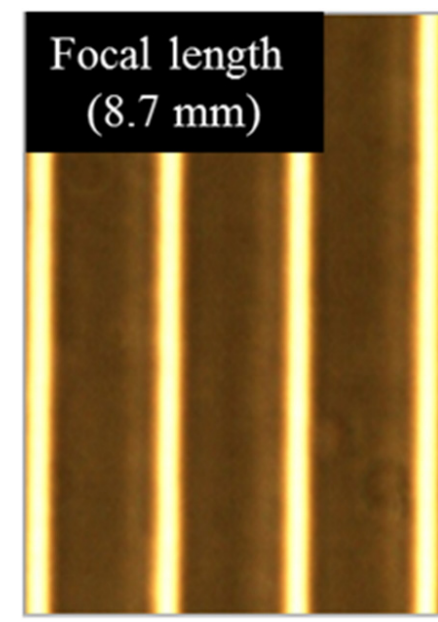

(a)

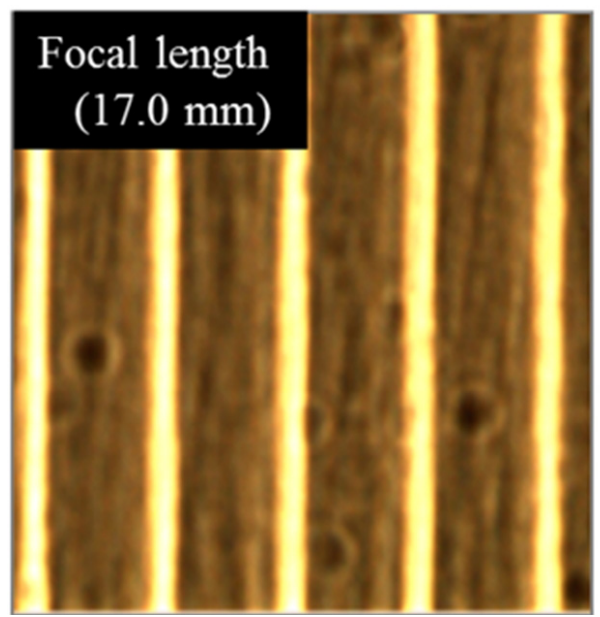

(c)

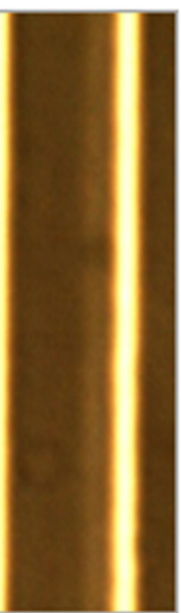

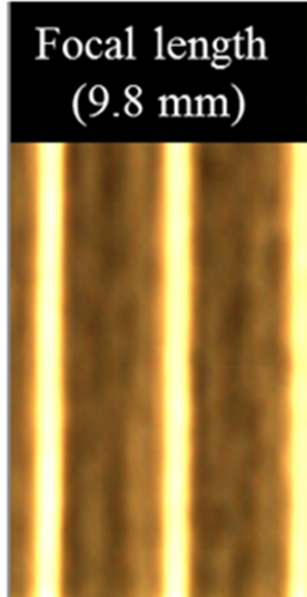

(b)

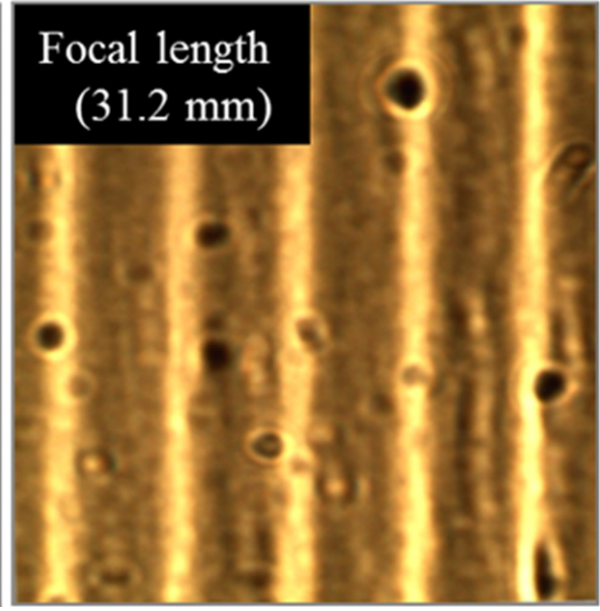

(d)

FIG. 3. Optical microscopy images of focused beams as a function of the heating temperature for the "off"-state of the TN cell. (a) $25^{\circ} \mathrm{C}$, (b) $40^{\circ} \mathrm{C}$, (c) $51^{\circ} \mathrm{C}$, (d) $56^{\circ} \mathrm{C}$. 


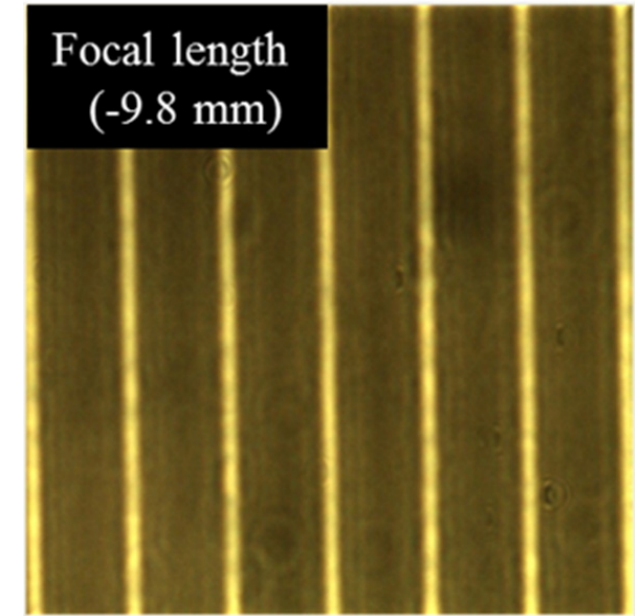

(a)

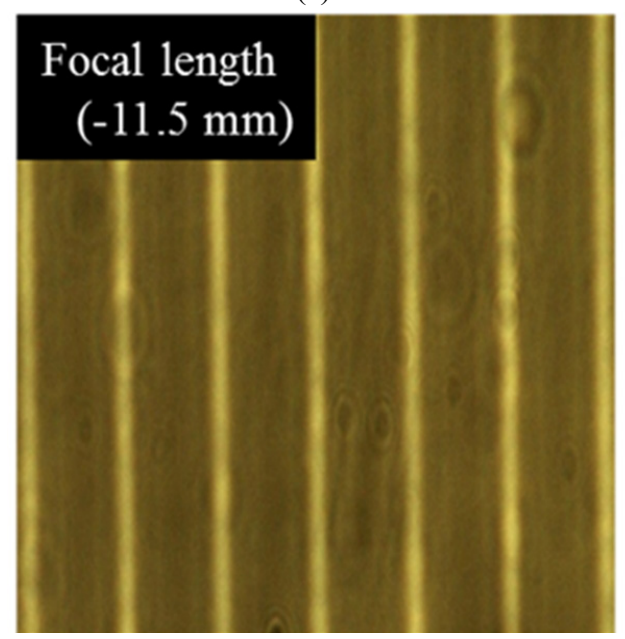

(c)

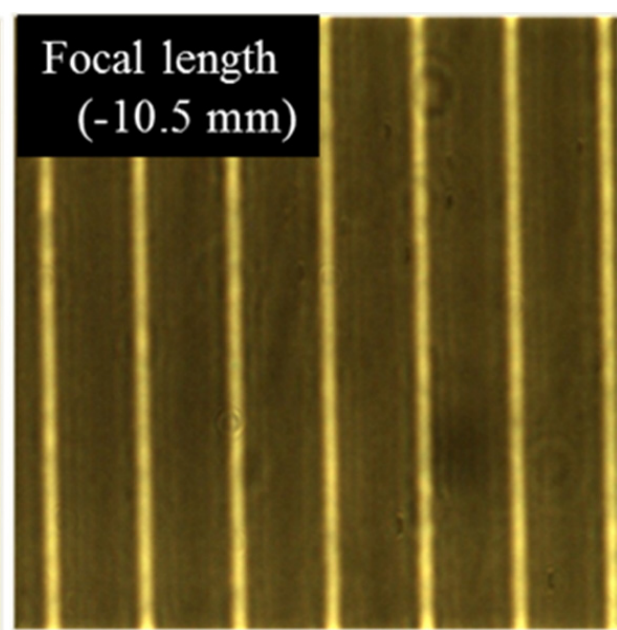

(b)

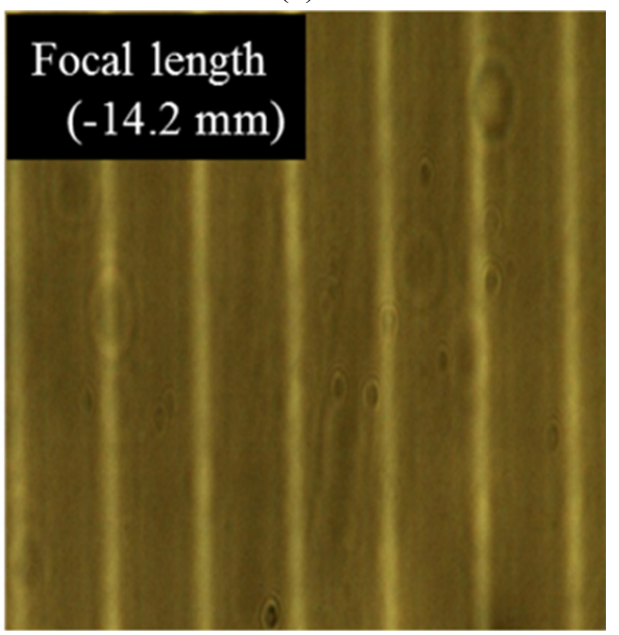

(d)

FIG. 4. Optical microscopy images of focused beams according to the heating temperature when the TN cell is in the "on"-state. (a) $25^{\circ} \mathrm{C}$, (b) $40^{\circ} \mathrm{C}$, (c) $51^{\circ} \mathrm{C}$, (d) $56^{\circ} \mathrm{C}$.

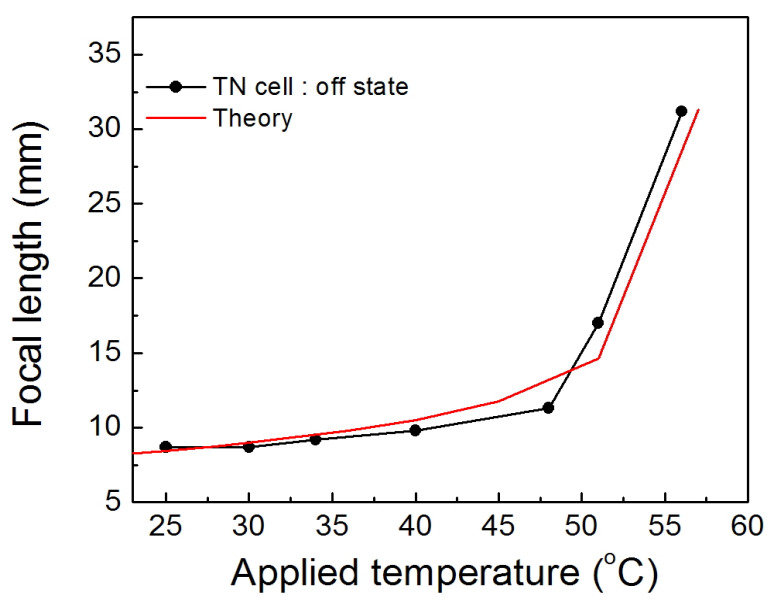

(a)

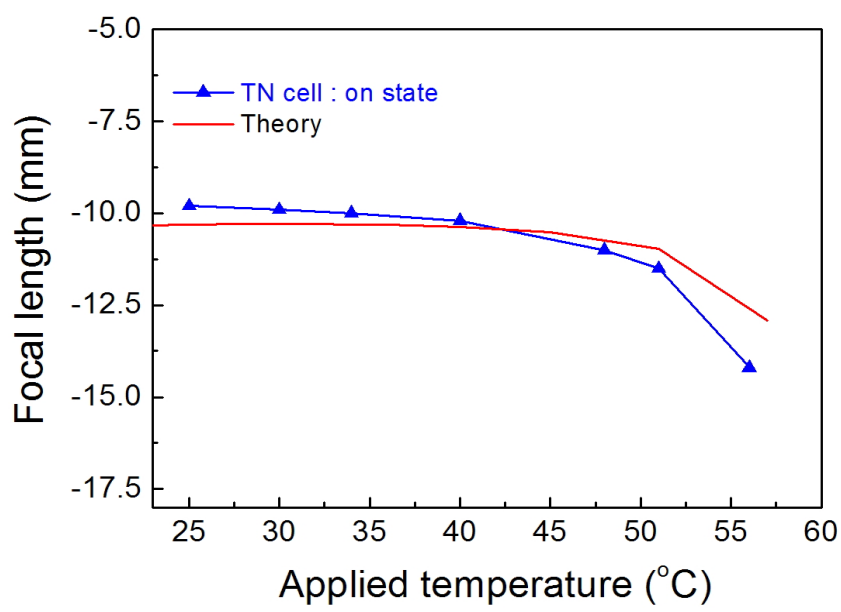

(b)

FIG. 5. Temperature-dependent focal length of the lenticular LC lens cell: (a) "off"-state, and (b) "on"-state of the TN cell. Black-circles represent the experimental data, while the red-solid line is the theoretical curve calculated using both Eq. (1) and the refractive indices of the E7 LC. 
by the lenticular LC lens at different temperatures controlled by the voltage applied to the transparent electrode in the lenticular LC lens cell. The focal length was varied from 8.7 to $31.2 \mathrm{~mm}$ with increasing temperature from 25 to $56^{\circ} \mathrm{C}$. These results suggest that the LC lens array functions well as a dynamic converging lens via temperature control.

The focal images at different temperatures when the TN cell was in the "on"-state were also observed to illustrate LC lens array acting as a dynamic diverging lens. Figure 4 shows the focusing properties of the beam through the lenticular LC lens cell. As estimated from Eqs. (3) and (4), the focal length of the beam images in the lenticular LC lens cell changed only slightly from -9.8 to $-11.5 \mathrm{~mm}$ below $51^{\circ} \mathrm{C}$ but increased in magnitude at normal level for temperature higher than $51^{\circ} \mathrm{C}$, reaching $-14.2 \mathrm{~mm}$ at 56 ${ }^{\circ} \mathrm{C}$. These results also indicate that the LC lens array can function well as a dynamic diverging lens via temperature control.

Finally, to determine the agreement between the experimental and theoretical results for the characteristics of the temperature-dependent focal length of the proposed lenticular LC cell, the experimental focal lengths were compared to the theoretical ones by plotting curves. Figure 5 shows the experimental results and the theoretical values calculated using Eq. (1) with the temperaturedependent refractive indices of $\mathrm{E} 7 \mathrm{LC}$ reported in previous studies $[12,18]$. The focal length of the lenticular LC lens was measured by refocusing the dimmed image at each temperature adjusted by Joule heating with an applied voltage. As shown in Figs. 5 (a) and (b), the red line indicates the theoretical plot evaluated from Eq. (1) with the refractive indices reported in previous studies according to temperature using Wu's model. The experimental results showed some agreement with the theoretical values.

\section{CONCLUSION}

A thermally controlled lenticular lens array was produced using a UV-curable curvature polymer structure filled with LC combined with a TN cell, and its optical properties were investigated. The TN cell makes the LC lens function as a diverging or converging lens by electrically controlling the polarization of incident light entering through a polarizer. The focal length of the lenticular LC lens could be tuned continuously by changing the effective refractive index of the LC via Joule heating for each lens type. Consequently, the focal length of the lens with the E7 LC was changed continuously from 8.7 to $31.2 \mathrm{~mm}$ for a converging lens type under the "off'-state of the TN cell, and from -9.8 to $-14.2 \mathrm{~mm}$ for a diverging lens under the "on"-state of the TN cell when the temperature was increased from 25 to $56^{\circ} \mathrm{C}$. The proposed LC lens may be limited in efficacy by the surrounding environmental temperature. As an alternative, we could choose a liquid crystal suited to ambient climatic characteristics.

\section{ACKNOWLEDGMENT}

This study was supported by a 2014 Yeungnam University research grant and the Human Resources Development Program (No. 20124030200100) of the Korea Institute of Energy Technology Evaluation and Planning (KETEP) grant funded by the Korea government Ministry of Trade, Industry and Energy.

\section{REFERENCES}

1. M. Sluijter, A. Herzog, D. K. G. de Boer, M. P. C. M. Krijin, and H. P. Urbach, "Ray-tracing simulations of liquidcrystal gradient-index lenses for three-dimensional display," J. Opt. Soc. Am. B 26, 2035-2043 (2009).

2. N. A. Riza and M. C. Dejule, "Three-terminal adaptive nematic liquid-crystal lens device," Opt. Lett. 19, 1013-1015 (1994).

3. T. Nose, S. Masuda, S. Sato, J. Li, L. C. Chien, and P. J. Bos, "Applications of liquid crystal to variable-focusing lenses," Opt. Lett. 22, 351-353 (1997).

4. H. Ren and S. T. Wu, "Tunable electronic lens using a gradient polymer network liquid crystal," Appl. Phys. Lett. 82, 22-24 (2003).

5. W. Wang, J. Fang, and K. Varahranmyan, "Nature of subpicosecond terahertz pulse propagation in practical dielectricfilled parallel-plate wave-guides," IEEE Photon. Technol. Lett. 17, 2643-2645 (2005).

6. S. Masuda, S. Takahashi, and T. Nose, "Liquid-crystal microlenses with a beam-steering function," Appl. Opt. 36, 4772-4778 (1997).

7. W. Zhang, K. Aljasem, H. Zappe, and A. Seifert, "Completely integrated, thermo-pneumatically tunable microlens," Opt. Lett. 19, 2347-2362 (2011).

8. S. Grilli, L. Miccio, V. Vespini, A. Finizio, S. De Nicola, and P. Ferraro, "Liquid micro-lens array activated by selective electrowetting on lithium niobate substrates," Opt. Lett. 16, 8084-8086 (2008).

9. H. W. Ren and S.-T. Wu, "Tunable-focus liquid microlens array using dielectrophotoretic effect," Opt. Express 16, 26462652 (2008).

10. H. W. Ren, S. Xu, Y. F. Liu, and S.-T. Wu, "Switchable focus using a polymeric lenticular microlens array and a polarization rotator," Opt. Express 21, 7916-7925 (2013).

11. S. Sato, "Liquid-crystal lens with variable focal length," Jpn. J. Appl. Phys. 18, 1679-1684 (1979).

12. J. S. Gwag, I.-Y. Han, C.-J. Yu, H. C. Choi, and J.-H. Kim, "Continuous viewing angle-tunable liquid crystals display using temperature-dependent birefringence layer," Opt. Express 17, 5426-5432 (2009).

13. J. Li, S. Gauza, and S.-T. Wu, "Temperature effect on the liquid crystal refractive indices," J. Appl. Phys. 96, 19-24 (2004).

14. Y. S. Choi, J.-H. Park, J.-H. Kim, and S.-D. Lee, "Fabrication of a focal length variable microlens array based on a nematic liquid crystal," Optical Materials 21, 643-646 (2002).

15. J. Li, S.-T. Wu, S. Brugioni, R. Meucci, and S. Faetti, 
"Infrared refractive indices of liquid crystals," J. Appl. Phys. 97, 073501-503 (2005).

16. H.-S. Ji, J.-H. Kim, and S. Kumar, "Electrically controllable microlens array fabricated by anisotropic phase separation from liquid-crystal and polymer composite materials," Opt. Lett. 28, 1147-1149 (2003).

17. J.-H. Kim and S. Kumar, "Fast switchable and bistable microlens array using ferroelectric liquid crystals," Jpn. J.
Appl. Phys. 43, 7050-7053 (2004).

18. K. C. Heo, S. H. Yu, J. H. Kwon, and J. S. Gwag, "Thermally tunable focus lenticular-lens using liquid crystal," Appl. Opt. 52, 8460-8464 (2013).

19. J. Li, G. Baird, Y.-H. Lin, H. W. Ren, and S.-T. Wu, "Refractive-index matching between liquid crystals and photopolymers," J. Soc. Information Display 13, 1017-1026 (2005). 